\title{
Documentar as Artes. Entre dois Pólos: um Arquivo de Objetos e Papeis e um Arquivo Nativo digital
}

\author{
Documenting Arts. Between two Poles: an Archive of Paper and Objects and a Digital Born \\ Archive
}

Francesca Zanella

Presidente CSAC e Pro-reitora Assuntos Gerais e Legais e Coordenação de Atividades de Internacionalização Universidade de Parma, Itália. E-mail: francesca.zanella@unipr.it

\section{Resumo}

Através dessa contribuição, pretende-se enfrentar um âmbito bastante articulado e vasto como o dos arquivos, objetos de amplo debate, através de dois estudos de caso diferentes por história e natureza, o Centro Studi e Archivio della Comunicazione da Universidade de Parma - CSAC - e o MoRE, Museum of Refused and Unrealised Art Projects - http://www.moremuseum.org/omeka/. Dois arquivos dedicados à pesquisa artística e projetual contemporânea, no primeiro caso italiana, a partir das primeiras décadas de 1900, o segundo internacional e italiana, da segunda metade de 1900 e contemporânea. Os dois arquivos estão relacionados por um objetivo comum: a necessidade de conjugar a pesquisa sobre a contemporaneidade através da coleta e arquivamento de documentos do fazer artístico.

Palavras-Chave: Arquivos Digitais. Arquivos de Arte Visual. Práticas de Arte Contemporânea. Arte Italiana. Arte não realizada. Projetos.

\begin{abstract}
The essay intends to offer a contribution to the archival studies through two study cases that have different stories and diversified nature: the CSAC (Study Center and Research on Communication), and MoRE (Museum of Refused and Unrealised Art Projects - http://www.moremuseum.org/omeka/-.). These are two archives devoted to contemporary art and design practices. CSAC is mainly devoted to italian art and design of the XX century; MoRE is an archive of unrealised works of art from the second half of the XX century till today. CSAC is an archive of paper, while MoRE is a digital repository. Finally both the archives have a common objective: promoting the research on contemporary art practices through the collecting and cataloguing of works of art and items of the design process.
\end{abstract}

Keywords: Digital Archive. Visual Art Archive. Contemporary Art Practices. Italian Art. Unrealised Art Projects. 


\section{Dois estudos de caso: CSAC e MoRE}

É possível afirmar que a atenção reservada ao papel do arquivo a partir das últimas décadas do século XX é determinada, em primeiro lugar, pela pressão que as tecnologias digitais impuseram às práticas arquivistas e ao debate teórico. No âmbito das artes visuais e do projeto, por outro lado, a reflexão sobre o papel do arquivo foi, em alguma medida, solicitada pelas pesquisas de alguns artistas que individualizaram nos repositórios das memórias individuais e coletivas a fonte de suas próprias pesquisas. Trata-se de duas perspectivas muito diferentes. A primeira é a dos arquivistas, profissionais formados para gerenciar coleções de documentos, mediadores entre arquiteturas de informações e usuários; a segunda é a de uma tipologia outra, com uma abordagem epistemológica diferente face à dos arquivistas e historiadores, própria de quem adentra arquivos em quanto fontes para a memória, para criar novas narrativas (literárias, visuais, sonoras).

Com assa contribuição pretende-se enfrentar um âmbito tão articulado e vasto qual é o dos Archival Studies, objeto de amplo debate, através de dois estudos de caso diferentes por história e natureza, o Centro Studi e Archivio della Comunicazione da Universidade de Parma - CSAC - e o MoRE, Museum of Refused and Unrealised Art Projects http://www.moremuseum.org/omeka/-, todavia relacionados por um objetivo comum: a necessidade de indagar a contemporaneidade através da coleta e arquivamento de documentos do fazer artístico.

\section{CSAC}

O Centro Studi e Archivio della Comunicazione (CSAC) é um centro da Universidade dos Estudos de Parma, que passa a ser denominado dessa forma depois de um percurso ao longo do qual se individualiza a origem na organização por parte do então Instituto de História da Arte da primeira exposição dedicada ao artista de Bolonha Concetto Pozzati, com a curadoria de Arturo Carlo Quintavalle, em 1968, nas salas farnesianas do Palácio da Pilotta, no centro de Parma.

A história e as razões da criação de um centro dessa natureza foram em várias ocasiões esclarecidas pelo fundador Quintavalle, como pode ser analisado em CSAC (1983), Nizzoli (1989), Quintavalle e Bianchino (2010), Quintavalle (2005) mas, dessa vez, vamos tentar integrar e esclarecer essa história a partir do próprio ‘arquivo', em particular de alguns entre os 
documentos produzidos na primeira década de vida (relações e atos administrativos), para dar conta das atividades desenvolvidas e para pedir o reconhecimento de uma realidade inédita que estava crescendo no âmbito do ateneu de Parma.

A natureza de laboratório é um dos aspectos mais significativos: a atividade expositiva que, desde 1968 se realiza constantemente, tornava-se possível com ritmo crescente pela instituição de um Centro de Exposições ao qual eram confiadas as produções dos ciclos expositivos que, em alguns casos, viram os próprios estudantes de história da arte diretamente envolvidos. A componente experimental colocava-se também na definição do campo de pesquisa: artistas que estavam se afirmando naqueles anos (da geração entre 35 e 40 anos) e, ao mesmo tempo, uma progressiva abertura para todas as formas da pesquisa visual (sátira, ilustração, mídia, fotografia), para marcar a recusa de qualquer hierarquia entre as artes. O nome 'Centro Studi e Archivio della Comunicazione' definiu-se exatamente para enunciar, sem ambigüidade, qual era o projeto cultural no qual se embasava a atividade: reconduzir a pesquisa artística no âmbito do sistema das imagens e da comunicação. As referências culturais podem ser retraçadas no debate crescente nacional e internacional sobre comunicação, sobre as chamadas novas mídias, em particular na linguística e na semiótica ${ }^{1}$.

Existe, todavia, uma outra vertente que é inseparavelmente ligada à definição do campo de pesquisa, e é a do debate sobre museu. Quando, em 1975, o conselho de administração da Universidade de Parma institui o Centro de Estudos e Arquivo sobre Comunicação Visual, engloba, em um organismo único o Centro de Exposição de Arte Contemporânea e o Centro de Estudos e Arquivo da Fotografia. Nas relações que acompanham esse processo, o termo museu, todavia, ainda continua a ser utilizado, quase para marcar a necessidade de acompanhar uma importante transição, permanecendo ancorados a um modelo adquirido, para facilitar a compreensão e a natureza de uma realidade inédita que estava crescendo no âmbito do ateneu. É evidente que, mesmo falando em museu, o modelo que se pretende realizar é o de uma instituição ou, melhor, de um contexto, em que a relação entre obras e público se funda na tensão continua rumo à compreensão da realidade da qual as obras são testemunhas. O público entra no museu, que é um arquivo, pois a estrutura espacial e as modalidades de fruição são impostadas de maneira a induzir o estudioso ou o visitante à compreensão dos fenômenos expressivos ou dos projetos e das linguagens. Não se trata, unicamente, de uma crítica ao 'White Cube', como apresentado por Brian O’Doherty nos artigos publicado em 1976 “Art Forum”,

\footnotetext{
${ }^{1}$ Desde a linguística estrutural de De Saussurre, até os ensaios sobre as estruturas dos contos de fadas de Propp, até os ensaios de Umberto Eco e Roland Barthes, são essas as principais referências.
} 
em seguida reunidos em Inside the white Cube. The ideology of the gallery space (2000) mas de uma crítica à estrutura do conhecimento.

Nesse caso, também, são inúmeras as referências ao debate e aos acontecimentos contemporâneos: desde a crítica por parte dos artistas perante às instituições artísticas que tiveram na Institutional Critique uma das expressões mais significativas desde os anos de 1960, até as contestações que, durante 1968, explodiram envolvendo e arrastando os museus e os ciclos expositivos nacionais e internacionais (ALBERRO; STIMSON, 2009) ${ }^{2}$. Nesses mesmos anos, aliás, a atenção para com o papel do artista, para com a definição de sua margem de liberdade em relação ao crítico, ao mercado e ao museu, é ladeada por uma consciência maior da necessidade de pesquisar uma centralidade renovada do espectador. A história do projeto cultural e arquitetônico do Centre Georges Pompidou de Paris representa, a esse respeito, uma das passagens fundamentais do debate sobre o papel do museu. A esse respeito, um dos textos de destaque é o estudo de Bourdieu e Darbel (1966), além do bem conhecido texto de Baudrillard (1977). Ao debate italiano e internacional sobre o novo Centre Pompidou é dedicado o livro de Martinelli (1978).

Existe uma ulterior passagem na história do CSAC que define a estrutura do centro/arquivo assim como chegou até os dias atuais. Em 1979, nos Estábulos do Palácio da Pilotta, inaugura-se a exposição dedicada a Bruno Munari, a primeira que inicia a atividade do recém fundado Departamento Projeto, que começa a crescer graças às doações de protagonistas do debate sobre o desenho industrial, sobre a gráfica e, ainda, sobre a moda, bem como da produção italiana contemporânea (Enzo Mari, Rosselli, Giancarlo Iliprandi, Achille Castiglioni, Brunetta, as Irmãs Fontana). A reflexão sobre o papel do desenho de projeto investe as esferas da pesquisa histórica, da análise do projeto, do método de trabalho e, finalmente, mas não último, da preservação dos arquivos de arquitetos e designer: são esses os temas sobre os quais se discute na ocasião do encontro nacional promovido pelo centro da Universidade de Parma (ARGAN, 1983), em anos em que, por exemplo, a própria Trienal de Milão desenvolve um novo programa cultural através do qual pretende-se renovar o papel da instituição lombarda que, desde a década de 1920, promovera o debate sobre projeto (ZANELLA, 2012).

\footnotetext{
2 Esse debate, na Itália, era conduzido em revistas como Nac, fundada em 1968 por Francesco Vincitorio e Data, fundada em 1971, idealizada e conduzida por Tommaso Trini.
} 
Um dos questionamentos é sobre qual é o posicionamento do CSAC no limiar da década de 1970 em relação ao debate sobre arquivo e, principalmente, em relação a qual debate sobre arquivo.

Nas inúmeras testemunhas de Arturo Carlo Quintavalle é explícita a referência à tradição dos Annales (LE GOFF, 1978; QUINTAVALLE, 1988) e à cultura antropológica francesa; fala-se de "Arquivo dos materiais, portanto não simplesmente de documentos, mas sim daquilo que os historiadores chamam 'monumentos', arquivo, portanto, não unicamente dos textos escrito, mas de cada texto, construído com cada 'escrita', literária, bem como de imagem, e com todo instrumento de transcrição, manuscrito bem como mecânico, desde a gráfica, até a fotografia, a TV, o filme" (CSAC, 1983, p. 9). Que se trate de referências ligadas ao debate historiográfico que se desenvolve com base na arqueologia do saber de Foucault (1969) e dos ensaios de Le Goff $(1974 ; 1988)$ é plenamente coerente com o contexto no âmbito do qual acontece a construção do arquivo, entendido como instrumento de pesquisa: a Universidade.

O termo 'arquivo', com efeito, não é assumido tendo em conta as definições dadas dentro da disciplina da arquivologia, em vários sentidos limitadoras:

1. O conjunto de documentos produzidos e adquiridos por uma entidade (sujeito produtor) no exercício de suas funções;

2. O local de preservação dos documentos;

3. O instituto de preservação, a entidade de natureza principalmente cultural, que se ocupa da preservação da documentação.

Essa perspectiva assume, dessa maneira, um papel central, portanto inelutável, no momento em que a atividade de coleta e de estudo por parte do centro se estende ao amplo contexto do projeto (arquitetônico, urbanístico, de design industrial, de gráfica e de moda). Trata-se de um importante ponto de virada na história do centro, porque reforça a ideia de arquivo enquanto conjunto de documentos, monumentos, vestígios da história, derrubando as hierarquias tradicionais, permitindo leituras sincrônicas e diacrônicas de maneira ainda mais significativas em relação a como acontecera até aquele momento, com as coleções das seções de arte, mídia e fotografias.

Além disso, a aquisição de arquivo de projetos impõe uma reflexão, sim, sobre o método, mas também sobre a configuração dos espaços e sobre a relação entre o arquivo e o 
contexto urbano. A dialética entre o momento expositivo, entendido como condição absoluta de abertura ao público, e o do arquivamento no interior da sede do Instituto de História da Arte nos contrafortes do Palácio da Pilotta, com efeito passa a faltar a partir do momento em que se institui um Departamento que nada mais é do que um arquivo que retende se abrir para a consulta, a pesquisa, a reflexão. É um processo que se desenrola ao longo de uma década, durante a qual o complexo inteiro do Palácio da Pilotta se torna objeto de um projeto de redefinição dos percursos expositivos embasados no projeto do arquiteto Guido Canali. O arquivo da comunicação contemporânea convive, de fato, com instituições como a Biblioteca Palatina, o Museu Arqueológico, a Galeria Nacional, o Teatro Farnese, o Instituto de Arte Toschi e a Universidade: uma verdadeira fortaleza da cultura. Esta é a história inicial que se desenvolveu até agora, levando à aquisição de 12.000.000 de objetos e desenhos que encontraram, em seguida, uma colocação no interior de um prédio industrial (Pavilhão Nervi) e, desde 2007, no interior da Abadia de Valserena. Face ao projeto da década de 1970 mudaram os pressupostos culturais, o debate sobre museu, o papel dos museus no âmbito das Universidades, pedindo, agora, uma reflexão sobre as políticas de aquisição, as problemáticas de preservação e, acima de tudo, de comunicação e divulgação através de ações múltiplas. Cresceu, na Itália também, o cuidado para com os arquivos 'visuais' e de projetação, como documentado pela série de encontros promovidos pelo ASAC, Arquivo Storico delle Arti Contemporanee da Bienal de Veneza, instituto inaugurado exatamente em 1976 na nova sede do Palácio Corner da Rainha, recolhendo a herança do Arquivo Histórico da arte contemporânea ${ }^{3}$ anterior.

\footnotetext{
3 I convegni internazionali, giunti alla terza edizione sono dedicati al rapporto tra Archivi ed esposizioni (Archivi e mostre) ed hanno avuto inizio nel 2013.
} 


\section{Arquivo e Prática arquivista}

Voltamos, portanto, à definição do termo arquivo acima colocada: o arquivo è, ao mesmo tempo, o conjunto dos documentos, o lugar que os acolhe e a instituição preposta à coleta e gestão deles. Um questionamento que, até agora, não foi colocado em relação à história do CSAC, é o do papel que a atividade de ordenação e catalogação possa ter tido para um centro universitário dessa natureza na potencialização de suas atividades coma as finalidades de pesquisa e de didática e na definição de um modelo alternativo de museu.

Já verificamos quanto nas declarações de intenção assumir o modelo de arquivo em detrimento do de museu fosse o sinal da vontade de propor modalidades alternativas de relação entre os documentos/monumentos e o público, rejeitando o modelo modernista, fundado na autonomia da obra de arte, sacralizada no templo das artes.

Não podemos esquecer o fato de que, naquela época (década de 1970 e de 1980), as leituras inovadoras sobre o papel do arquivo provinham da filosofia e da historiografia. A arquivologia permaneceu ligada a uma definição do papel do arquivista enquanto curador de pegadas documentárias derivadas ou conectadas às atividades de organizações, indivíduos, famílias, empresas ou outros grupos sociais, resguardando o contexto significante, o propósito e a função originais do documento. Em relação ao desenvolvimento da disciplina na Itália ao longo do século XX, alguns entre os textos mais relevantes são os escritos de Abati (1937), Lodolini (1955), Briguglio (1958). O resguardo da relação dos papeis, a necessidade de atribuir valor científico e objetivo da atividade do arquivista leva também à formulação das descrições e de todos os instrumentos de ordenação do conjunto dos documentos em função de sua acessibilidade e valorização. È importante, todavia, lembrar que, perante uma continuidade também nas décadas seguintes na afirmação do papel central do contexto, definido pelo conceito de vínculo. Em relação a isso, remetemos a Horsman (1994) e a Winget (2005).

O que mudou, hoje, na teoria arquivista em relação ao significado atribuído ao documento desde a teoria positivista para frente enquanto testemunha da intenção do autor, e em relação à necessidade de devolver com a maior objetividade essa intenção, é exatamente a visão fundada na ideia da objetividade do método de ordenação e descrição e, conseqüentemente, o significado que deve ser atribuído ao documento. $\mathrm{O}$ arquivista, em alguns casos, é assim definido um performer, deslocando o acento da análise do sistema de documentos para a indagação dos processos que constituíram a acumulação de documentos, 
como discutem Eastwood (1994), Valderhaug [20??] e Cook e Schwartz (2002).

Essa mudança é, também, ladeada pela acrescida consciência da modificação que a aplicação dos instrumentos de catalogação eletrônica e as possibilidades de estruturação e disseminação das informações pelo world wide web introduziram na teoria e na prática de arquivo. É exatamente nos últimos anos, com efeito, que se desenvolve a reflexão acerca do dinamismo dos arquivos, e acerca do arquivo enquanto processo e não mais unicamente enquanto expressão, fotografia e cristalização da atividade de uma instituição ou de um indivíduo (NOORDERGRAAF, 2011).

O Centro Studi e Archivio della Comunicazione, na primeira fase de atividade, utiliza a descrição, permanecendo no âmbito da tradição da história da arte, de um lado desenvolvendo, em primeiro lugar, a pesquisa crítica, através da redação de textos e fichas analíticas para os catálogos das exposições, de outro lado entendendo o fichamento, enquanto registro dos componentes que constituem o documento, portanto o produto do fazer artístico, como simples instrumento de gestão e acesso aos documentos coletados no arquivo (inventário).

A comparação com os padrões internacionais de catalogação e com o debate sobre o papel dos arquivos de arte e do projeto acontece de forma mais sistemática somente depois da metade da década de 1980, sem que isso incida de maneira significativa sobre as atividades de gestão e sobre a reflexão do arquivo na era do world wide web.

Todavia, não se pode negar que o CSAC, cujas coleções até hoje alcançaram uma dimensão única dentro do panorama nacional bem como em nível internacional como lugar de documentação da cultura italiana do século XX, constitui um caso de estudo significativo face à relação entre pesquisas sobre projetos e visuais e arquivo. Esta consciência está na base do novo projeto, lançado em 2014, para uma valorização do patrimônio, para uma reinterpretação do papel de tamanha coleção no âmbito do debate atual e, também, para uma impostação de um programa de catalogação e de um plano digital. 


\section{Arquivo, Museu, Plataformas digitais e Pesquisas artísticas}

Já é comumente aceito que o ensaio de Hal Foster (2004) constitui a primeira análise da prática de trabalho sobre arquivos por parte de alguns artistas dando, de alguma maneira, a partida para um aumento de aprofundamentos que ampliaram a pesquisa sobre práticas artísticas contemporâneas no âmbito dos Museum Studies, com um foco na relação entre museu e arquivo. Outros autores que se dedicam ao tema são Merewether (2006), que mapeia os pressupostos teóricos e as experiência dos artistas e curatore europeus face a relação com os arquivos; lembramos, também, do simpósio de 2007 The archival impulse: artists and archives, cujo título se origina na contribuição de H. Foster; em 2009 foi promovido o simpósio Archiving the artist, cujo foco foi o arquivo dos artistas e cujos resultados se encontram nos Tate papers, organizados por Sue Breakell, arquivista do Tate; enfim, lembramos Archiving the artist, organizado por Vaknin, Stuckey e Lane (2013), resultado de um segundo encontro dedicado à análise da relação entre artista e arquivo.

São esses os pressupostos sobre os quais a reflexão atual sobre o futuro do CSAC se fundamenta: o grande arquivo è, com efeito, testemunha a $360^{\circ}$ da cultura do projeto italiano, e pode ser entendida como uma plataforma para a reflexão atual de artistas e designers.

Esses são, também, os pressupostos do outro projeto aqui proposto, o MoRE Museum que, centrado no tema do que não foi realizado, escolheu o caminho do digital, colocando-se dentro de um contexto preciso, que é o dos arquivos digitais que, em inúmeros casos, estão se tornando os instrumentos de trabalho, os meios expressivos de artistas e curadores.

Mais uma vez, è necessário voltar para as múltiplas definiç̧ões de arquivo que apontam tanto o conjunto dos documentos, quanto o lugar que os hospeda, bem como a instituição. No contexto das Digital Humanities, a reflexão renovada de tais definições, bem com, também, sua simples aceitação requer, de qualquer maneira, um questionamento sobre quais são os efeitos e as mudanças ligados à tradução digital dos documentos analógicos, e quais as mudanças sobre a maneira de transmitir a memória e indagar no passado em uma sociedade cada vez mais condicionada pela comunicação digital, mas que, acima de tudo, produz conteúdos e documentos nativos digitais, sem esquecer o surgimento da crise de uma ideia de tempo linear em forte contradição com o sistema de produção e transmissão das informações e dos documentos. São estes, com efeito, os questionamentos que formulam, hoje, historiadores, 
críticos, digital humanists, bem como artistas, instituições e indivíduos produtores de conteúdos.

O debate já è bem amplo e ainda difícil de ser circunscrito, ainda porque ultrapassa os contextos tradicionais da arquivologia, da historiografia, da história da arte e da ciência da informação. Podemos, nessa sede, iluminar alguns conceitos particularmente significativos.

Queremos começar pela contribuição sobre arquivos audiovisuais de Julia Noordegraaf (2011) que, refletindo sobre as afirmações de Rick Prelinger (2009), fundador, em 1996, do grande arquivo online archive.org (https://archive.org/about/), a propósito da mudança que as coleções online provocaram nas expectativas dos usuários face esses recursos, que estejam, disponíveis, de acesso livre e participativas, destaca que, apesar das instituições ainda possuir a função de gatekeepers principais, particularmente pelos direitos de copyright, a extensão de arquivos virtuais mudou a relação entre usuários e aqueles que têm a posse dos documentos, tornando o papel do aquivista, entendido como o principal detentor do conhecimento do arquivo, um desafio.

Perante tais perspectivas, próprias de quem opera dentro de instituições tradicionalmente deputadas à conservação e gestão da memória, o de quem se pergunta a respeito do papel dessas instituições, é interessante considerar também as reflexões sobre a natureza dos arquivos digitais. Como aquelas de Wolfgang Ernst, teórico das mídias que, em um ensaio de $2016^{4}$, fala acerca do dinamismo dos arquivos digitais que se origina, em primeiro lugar, pelo fato de que os objetos são definidos por um código binário, e o processo de digitalização muda também o próprio arquivo. O foco, portanto, não é mais somente na estrutura, no conteúdo, mas acima de tudo nas relação. O discurso de Ernst inicia pela análise dos arquivos administrativos, caracterizados pela ausência de narrativa que o processo de digitalização, por outro lado, poderia introduzir:

Beyond the "cultural turn" of the last two or three decades concerned with cultural and collective memory, the critical focus has now shifted to the analysis of technocultural temporal dynamics of social, administrative and technological systems. The archive is set in motion. Let us therefore address the archive not as a coherent depository for memory supply but instead identify its multiplicity of temporal layers with and within memory technologies. Since the notion of the archive has been

\footnotetext{
4 Wolfgang Ernst, Unfolding the Layers of the 'colonised' archive, in Decolonisign archives, L'Internationale .., p. 10: "In cultural discourse, in the art world and in political activism, the term "archive" has mostly become a generalised metaphor for different kinds of collections of traces from the past. While in public discourse the archive is mostly (mis-) understood as the "content" of the archive (its records, its data banks), in archival sciences the term rather refers to the organising structure. Against intellectual or artistic fantasies of "the anarchival" (Fürlus \& Giannetti 2014), the digital archive is still rigorously rooted in its techno-mathematical structure, while the dynarchive lies between the archival and the anarchival spheres."
} 
extended from the symbolic order (alphabetical texts) to the storage of signals (like physical sound and imagery), a memory has emerged which is capable of addressing human perception in a kind of repeatable hyper-presence. This does not only represent, but actually enacts different aggregations of the past. My epistemological intention is to liberate archival memory from its reductive subjection to the discourse of history and re-install it as an agency of multiple temporal poetics in its own right" (ERNST, 2016).

Ernst (2010) reflete contemporaneamente sobre a dimensão temporal do arquivo digital, sobre as possíveis formas de resistência em reivindicar seu papel de monumento institucional, retendo o fluxo da comunicação eletrônica, sobre sua dimensão efêmera, multissensorial, correspondente à contemporânea cultura dos usuários menos cuidadosos e sensíveis à eternidade dos documentos e dos registros.

O debate é, de qualquer maneira, extremamente articulado, e torna-se complexo pela permanência da perspectiva da arquivologia clássica, da presença, contemporaneamente, de arquivos nativos digitais e de arquivos tradicionais traduzidos para o código binário, pela necessidade de reinterpretar conceitos quais os de vínculo de arquivo, mais do que de descrição, de reinterpretar o papel do arquivista e do arquivo em quanto instituição, mas também como lugar físico.

Se a teoria das mídias introduz à análise dos repositórios de textos e imagens no âmbito da rede, os instrumentos de interpretação das novas formas expressivas multicanais, dentro do pós-estruturalismo e dos Cultural Studies é retomada a temática da natureza do arquivo pós foucaultiano. Como acontece nas contribuições de Manuel de Landa, que promove uma reflexão nessa direção em um volume no âmbito do qual o antropologo Arjun Appadurai afirma que o arquivo, depois da contribuição de Foucault, "returns to its more general status of being a deliberate site for the production of anticipated memories by intentional communities" (APPADURAI, 2013, p. 13), comunidades internacionais que são aquelas ativas na rede, produtoras de conteúdos.

Fundamental é, também, a contribuição de Jeffrey Schnapp (2012), que insiste na necessidade de compreender a natureza do arquivo digital, de um lado, e de 'agredir' o arquivo (analógico ou digital que seja) transferindo-o da condição de patrimônio enterrado àquela de bem 'reativado'. Para alcançar esse objetivo, os caminhos são multíplices, contínuas são as experiências fundamentadas em uma ideia de descrição e visualização dos diferentes objetos da memória coletiva que potencialize sua natureza relacional e a natureza participativa dos arquivos do presente. 
Como interage o arquivista com esse contexto em que são minados os papeis tradicionais da produção documentária e em que a componente descritiva (metadados) se torna componente inseparável do próprio documento? Theimer (2014) sinaliza a necessidade de considerar o contexto de produção, quem e como criou os metadados dos arquivos digitais. Analiza varios casos de arquivos digitais, desde arquivos que espelham arquivos existentes, tradicionais, arquivos digitais que coletam materiais originários de contextos diferentes, até arquivos de objetos nativos digitais.

The value of the collections of materials preserved in archives often lies in the relationship of the records to each other - what's called the archival bond - which means that the whole is greater than the sum of the parts. As a whole, the materials provide evidence about the activities of their creator or the person or organization who brought them together ... this "remediation" of archival materials into a new form is also a tremendous opportunity to consider the past with more sensitivity, to pay far greater attention to how we access and analyze history itself. (THEIMER, 2014)

O que significa fazer história através de arquivos tão complexos e estratificados de textos e metatextos? Como pode mudar a pesquisa no interior de inéditos reticulados de informações aumentadas e de técnicas de visualização de objetos e de informações? Lev Manovich (2012), promotor do software Studies Initiative na Universidade da California, San Diego, por exemplo, ofereceu contribuições importantes em relação às técnicas de visualização e das análises computacionais aplicadas principalmente aos arquivos visuais, individualizando a contaminação entre o sistema de informação em rede da comunicação social face os recursos estruturados próprios da tradição das instituições culturais e de pesquisa.

São esses os questionamentos colocados como exemplos em 2014 na edição monográfica do "Journal of Digital Humanities":

The digital medium has challenged historians to expand their knowledge about archives, and understand their function in generating scholarship and knowledge. But what might be the key theoretical and methodological questions surrounding the intersection of digital archives or digital collections and historical practice? What materials do archives collect and preserve, and why? Which materials are selected, and which are excluded? What are the driving forces and principles guiding the contextual information about collections provided by archives? Which political, social, economic, and cultural power relationships now structure the archives? How do we cope with the sudden, and at times unexplained, disappearance of collections in digital archives (portions of American Memory being a prominent example, as one audience member suggested)? How important is contextualization of collections in the digital environment? How can archival metadata be better situated in place and in time? These are questions that archivists and historians might come together to confront in critical and productive ways (HERING et al., 2014). 


\section{MORE}

A esse contexto deve ser reconduzido o projeto MoRE (http://www.moremuseum.org/omeka/), um museu digital idealizado por Elisabetta Modena e Marco Scotti e inaugurado em 2012, nascido para refletir, através da individualização de projetos não realizados de artistas ativos desde a segunda metade do século XX, sobre o papel do artista, sobre os processos de produção, sobre as relações com a comissão e com o mercado. O museu digital, que se constitui por um repositório de arquivamento permanente, ligado a um site web, até hoje já chegou à sua segunda edição; com efeito, desde o primeiro site, realizado com WordPress, o time de curadores sentiu a exigência, depois de dois anos de atividades, de poder gerenciar de maneira mais avançada a relação entre as imagens e o aparado descritivo dos projetos. Depois de uma breve pesquisa no âmbito das plataformas open, foi adotada Omeka (http://omeka.org), um software open-source desenvolvido pelo Roy Rosenzweig Center for History and New Media, da George Mason University, com o qual se realizou um arquivo e uma interface de visualização dos materiais arquivados no repositório institucional DSpace da Universidade de Parma, que constitui o arquivo permanente como garantia do acesso aberto e da preservação permanente dos objetos digitais. Omeka consente, com efeito, de organizar as modalidades de visualização, criando também galerias virtuais através da indexação dos objetos digitais que são arquivados para cada projeto não realizado recolhido. A descrição de tais objetos, que são tanto o produto de digitalização de documentos em papel individualizados dentro do arquivo dos artistas, bem como objetos nativos digitais, acontece em níveis multíplices: através dos metadados Dublin Core e através de fichas de análise dos profeto individuais que acompanham os materiais.

Até hoje (maio de 2016), os artistas são 54 e os projetos registrados 90.

$\mathrm{Na}$ atividade de coleta de materiais de projetos, em seu arquivamento e descrição, a atenção do grupo de curadores, historiadores da arte e bibliotecários é voltada especificamente para duas temáticas: como analisar o trabalho do artista, como interagir com o artista na pesquisa no interior de seu arquivo em busca de projetos não realizados e como preservá-los, torná-los públicos e descrevê-los.

Para poder impostar uma análise comparativa entre os dois casos propostos nesse artigo é necessário lembrar da impostação da atividade de seleção dos artistas, do trabalho conduzido pelos curadores com eles para individualizar os projetos a serem arquivados. Com efeito, se o 
arquivo é o lugar da estratificação de pegadas e de entrelaçamento de ações das quais os documentos são marcas bem como produto, também para um projeto que se coloca como meta constituir um museu/arquivo das pesquisas artísticas contemporâneas o trabalho da curadoria deve ser analisado de maneira completa. Essa è, de fato, uma componente que distingue as duas experiências e que produz dois 'arquivos' diferentes, não somente pelos instrumentos utilizados. Diferentemente do arquivo CSAC, MoRE se constitui por testemunhas individuais de projetos não realizados, singulares ou, de qualquer maneira, parciais: trata-se, portanto, de uma retirada no âmbito do arquivo pessoal do artista, que è realizado por ele mesmo (ou por um curador/arquivista no caso de arquivos históricos), com o qual o curador se confronta, gerando um êxito que é o produto de duas intenções. Às vezes, a seleção é feita unicamente pelo artista, que pode produzir o objeto digital, ou que demanda ao curador essa atividade; em outros casos a escolha surge do diálogo. Todas essas variações são também elementos significativos para quem queira analisar a maneira de operar do autor, sua relação com os documentos do próprio fazer. Ao mesmo tempo, também as diferentes tipologias de mídia e escritas constituem uma testemunha das diversas formas de operar dos autores, de seus projetos. Elementos, todos eles, que devem ser destacados pelo aparato descritivo dos objetos, tanto em nível de metadados quanto de fichas críticas.

A história do MoRE é ainda curta e não existe um distanciamento suficiente para historicizar o projeto que, porém, já foi apresentado em contextos diferentes ${ }^{5}$ (Fundação Bevilacqua La Masa, Bienal de Veneza, Museu do Novecento Milano, MSU Zagreb), enquanto em 2015 foi realizada a primeira exposição 'física', que constituiu uma das primeiras ocasiões de reflexão a respeito das categorias do não realizado.

Uma ocasião importante de confronto com o debate atual foi a inclusão do MoRE como caso de estudo para o número monográfico da revista "Archives and Records" (ZANELLA et al., 2015) dedicado aos arquivos de artes visuais. Nesse contexto, a perspectiva era a da disciplina da arquivística que requeria um enfoque sobre as diversas práticas e metodologias. Naquele fórum procurou-se, portanto, descrever a metodologia de trabalho, fundamentada na exigência de respeitar a natureza digital dos materiais coletados, de analisar os casos, verificados no tempo, de tradução para o digital de documentos analógicos, e a interação com a natureza do repositório digital open access individualizado para uma preservação de longo

\footnotetext{
5 A esse respeito, ver o número temático Per un museo del non realizzato. Pratiche digitali per la raccolta, valorizzazione e conservazione del progetto d'arte contemporanea, Ricerche di S/Confine, v. 3, 2014. Disponível em: 〈http://www.ricerchedisconfine.info/dossier-3/index.htm>.
} 
prazo de objetos digitais diversificados; enfim, de calibrar modalidades de descrição, com a criação de metatextos finalizados à pesquisa, com a granularidade da informação através dos metadados e, finalmente, com a categorização dos casos da falta de realização de um projeto de arte.

\section{Considerações finais}

Buscando, assim, uma conclusão, podemos dizer que CSAC e MoRE constituem um campeão exemplificativo da complexidade das perspectivas atuais do papel de arquivos, museus, coleções enquanto instrumentos para a pesquisa sobre as práticas artísticas e sobre o design contemporâneos. Em um nível de extrema simplificação, poderíamos propor esse esquema, que constitui o ponto de partida para análises ulteriores:

Csac:

- Um arquivo dedicado às artes e ao projeto italianos.

- Um arquivo 'analógico' de papeis e objetos que, agora, se abre à dimensão expositiva permanente.

- Um arquivo em que hoje prevalece o modelo da preservação.

- Um arquivo que hoje chegou à linha divisória entre a dimensão do acesso e compartilhamento e aquela do 'enterro'

More:

- Um arquivo digital dedicado a projetos não realizados de artistas italianos e internacionais.

- Um museu digital que, porém, não exclui formas tradicionais como aquela da exposição temporária.

- Um recurso digital disseminada na web em que prevalece a atividade de mediação entre produtor e usuário.

- Um arquivo em que é forte a atenção para com as problemáticas da preservação do digital.

- Um arquivo em que a dimensão do compartilhamento é mediada.

InCID: R. Ci. Inf. e Doc., Ribeirão Preto, v. 7, n. 2, p. 4-22, set. 2016/fev. 2017. 
Em relação às perspectivas comuns de desenvolvimento podemos apontar caminhos de:

- Potencializar o Arquivo como recurso projetado rumo ao futuro e ao mesmo tempo testemunha da memória.

- Animar o Arquivo, através de ações diferentes que considerem a dimensão material e digital do espaço do arquivo/museu.

- Individualizar o público apropriado e as modalidades de fruição.

- Conjugar pesquisa e participação (unir comunidades diferentes: intentional communities).

- Definir modalidades do crescimento do Arquivo.

- Arquivo como plataforma do confronto disciplinar.

Não há dúvidas de que os dois arquivo têm em comum perspectivas possíveis de desenvolvimento, sugeridas pelo debate e pela prática contemporâneos. A potencialização do arquivo em quanto recurso projetado para o futuro e, ao mesmo tempo, testemunha da memória, pode se realizar através de linhas diferentes de intervenção, que se abrem com a individualização de seus próprios públicos e das respectivas modalidades de utilização, até a colocação em jogo de atividades para animar o Arquivo, através de várias ações que considerem a dimensão material e digital do espaço do arquivo/museu. Ainda, para um arquivo enquanto plataforma do confronto disciplinar, devem ser conjugadas pesquisa e participação, juntando comunidades diferentes (intentional communities), e definindo também as modalidades de crescimento das coleções documentárias. 


\section{Referências}

AA.VV. Situazione 1983. Parma: Università degli Studi/CSAC, 1983.

ABATI, D. Archivio: ordinamento e funzionamento. Roma: Supergrafica Lolli, 1937.

ALBERRO, A.; STIMSON, B. (Org.). Institutional critique: an anthology of artists' writings. Boston: MIT Press 2009.

APPADURAI, A. Archive and aspiration. In: BROUWER, J.; MULDER, A. (Ed). Information is alive. Rotterdam: Nai, 2003.

ARGAN, G. C. Introdução. In: AA.VV. Il Disegno dell'architettura: incontri di lavoro, Parma 23/24 ottobre 1980. Parma: CSAC/Università di Parma, 1983.

BAUDRILLARD, J. Effet beaubourg: implosion et dissuasion. Paris: Galilée 1977.

BOURDIEAU, P.; DARBEL, A. L'amour de l'art: les musées et leur public. Paris: Minuit 1966.

BRIGUGLIO, L. Sul concetto di archivio. Roma: Istituto Poligrafico dello Stato, 1958.

COOK, T.; SCHWARTZ, J. Archives, records, and power: from (postmodern) theory to(archival) performance. Archival Science, v. 2, p. 171-185, 2002.

EASTWOOD, T. What is archival theory and why is it important?. Archivaria, v. 37, Spring 1994. Disponível em:

<http://journals.sfu.ca/archivar/index.php/archivaria/article/view/11991〉.

ERNST, W. Unfolding the layers of the 'colonised' archive, in decolonisign archives, l'internationale. 02 fev. 2016. Disponível em:

$<$ http://www.internationaleonline.org/research/decolonising_practices/50_radically_de_histor icising the archive decolonising archival memory from the supremacy of historical disc ourse>.

Cultural archive versus technomathematical storage. In: RØSSAAK, E. (Org.). The archive in motion: new conceptions of the archive in contemporary thought and new media practices. Novus: Oslo, 2010.

FOSTER, H. An archival impulse. October, n. 110, Fall 2004.

FOUCAULT, M. L’archéologie du savoir. Paris; Gallimard, 1969.

HERING, K. et al. Digital historiography and the archives. Journal of Digital Humanities, v. 3, n. 2, summer 2014. Disponível em: 〈http://journalofdigitalhumanities.org/3-2/digitalhistoriography-and-the-archives>.

HORSMAN, P. Taming the elephant: an orthodox approach to the principle of Provenance. In: THE PRINCIPLE of Provenance: report from the First Stockholm Conference on Archival Theory and the Principle of Provenance 2-3 September 1993. Stockholm: Svenska Riksarkivet, 1994. 
LE GOFF, J. Documento/Monumento. In: ENCICLOPEDIA EINAUDI, T-V, p. 38-48, Torino: Einaudi, 1978.

Faire de l'histoire: nouveaux problèmes, nouvelles approches, nouveaux objets.

Paris: Gallimard, 1974.

Histoire et mémoire. Paris: Gallimard, 1988.

LODOLINI, A. La creazione di un grande archivio: l'archivio nazionale d'Italia all'EUR. Roma: Istituto poligrafico dello Stato/Libreria dello Stato, 1955.

MANOVICH, L. Museums without walls, art history without names: visualization methods for humanities and media studies. 2012. Disponível em:

$<$ http://manovich.net/index.php/projects/museum-without-walls-art-history-without-namesvisualization-methods-for-humanities-and-media-studies $>$.

MARTINELLI, G. Il Centro Beaubourg a Parigi: macchina e segno architettonico. Bari: Dedalo, 1978.

MEREWETHER, C. (Org.). The archive. London, Boston: Whitechapel Gallery: MIT Press, 2006.

NIZZOLI, M. Archivi del progetto. Milano: Electa, 1989.

NOORDEGRAAF, J. Remembering the past in the dynarchive: the state of knowledge in digital archives. In: CONFERENCE MEDIA IN TRANSITION: unstable platforms: the promise and peril of transition, 7., 2011. [Abstracts and papers]. Boston: MIT, 2011. Disponível em: 〈http://web.mit.edu/comm-forum/mit7/papers/Noordegraaf.pdf>.

O'DOHERTY, B. Inside the white Cube: the ideology of the gallery space. Regents of the University of Califórnia, 2000.

PRELINGER, R. The appearance of archives. In: SNICKERS, P; VONDERAU, P. (Org.). The YouTube reader. Stockholm: National Library of Sweden, 2009. p. 268-274.

QUINTAVALLE, A. C. CSAC quarant'anni: 1'archivio del novecento. In:

BIANCHINO, G. NOVE100: arte, fotografia, architettura, moda, design. Milano: Kira, 2010.

Il palazzo dell'arte. Milano: Fabbri, 1988.

Politica della cultura, Università e strutture museali: il Centro Studi e Archivio della Comunicazione (CSAC) dell'Università di Parma. Annali di Storia delle Università italiane, v. 9, 2005. Disponível em:

<http://www.cisui.unibo.it/annali/09/testi/20Quintavalle testo.htm>.

SCHNAPP, J. Buried (and) alive. In: DECOLONISING the archive. L'internationale books, 2016. Disponível em:

< http://www.internationaleonline.org/research/decolonising_practices/52_buried_and_alive $>$.

THEIMER, K. Distinction worth exploring: 'archives' and 'digital historical representations. Journal of Digital Humanities, v. 2, n. 2, Summer 2014. Disponível em: 
$<$ http://journalofdigitalhumanities.org/3-2/a-distinction-worth-exploring-archives-and-digitalhistorical-representations $>$.

VAKNIN, J.; STUKEY, K.; LANE, V. (Org.). All this stuff: archiving the artist. London: Libri, 2013.

VALDERHAUG, G. Between practice and theory: some reflections on archival science, the archival professions and archival education. Depotdrengen. [20??]. Disponível em: $<$ https://depotdrengen.wordpress.com/between-practice-and-theory---some-reflections-onarchival-science-the-archival-professions-and-archival-education/>.

WINGET, M. It was just in the air, at any place...: the principle of provenance $\&$ modern archival systems. Unpublished paper, v. 7, March 2005. Disponível em:

$\langle$ http://www.unc.edu/ winget/research/DigitalProvenance.pdf $>$.

ZANELLA, F. et al. MoRE, an archive of signs and traces of artistic practices: creating a tool for research in contemporary art and curatorial practices. Archives and Records: the Journal of the Archives and Records Association, v. 36, n. 1, p. 56-70, 2015. DOI: 10.1080/23257962.2015.1015260.

ZANELLA, F. Esporsi: architetti, artisti e critici a confronto. Verona: Scripta, 2012. 